\title{
Optical Characterization of Nitrogen-Vacancy Centers Created by Proton Implantation in Diamond
}

\author{
M. Schabikowski ${ }^{a, *}$, A.M. Wojciechowski ${ }^{b}$, M. Mitura-NowaK ${ }^{a}$, M. MrózeK $^{b}$,

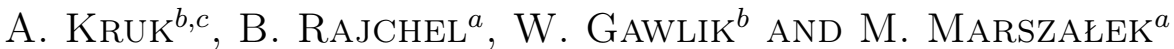 \\ ${ }^{a}$ Institute of Nuclear Physics, Polish Academy of Sciences, Kraków, Poland \\ ${ }^{b}$ Institute of Physics, Jagiellonian University, Kraków, Poland \\ ${ }^{c}$ Pedagogical University of Kraków, Institute of Technology, Kraków, Poland
}

\begin{abstract}
We report the results of high-energy $(1.8 \mathrm{MeV})$ proton implantation in a type-Ib diamond. The focused ion beam was used to introduce vacancies in a $20 \mu \mathrm{m}$-deep layer with applied doses in the range of $10^{13}$ to $10^{16}$ ions $/ \mathrm{cm}^{2}$. The sample was subsequently annealed in vacuum which resulted in migration of vacancies and their association with the nitrogen present in the diamond matrix. We studied properties of the formed nitrogen-vacancy centers with photoluminescence and optically detected magnetic resonance. The samples are aimed to be used in sensing applications, e.g., magnetometry and bio-magnetic imaging.
\end{abstract}

DOI: 10.12693/APhysPolA.137.9

PACS/topics: diamond, proton implantation, NV, color center

\section{Introduction}

The discovery of negatively charged nitrogen vacancy (NV) color centers in diamond [1] paved the way to many exciting applications, such as biophysics [2], quantum information [3], magnetometry [4, 5], and temperature sensing [6]. Due to their ground-state spin, $S=1$, and the spin-lattice interactions, NV centers can probe magnetic and electric fields, strain, pressure, and temperature with nanometer resolution when single color centers are used for sensing applications [7, 8]. On the other hand, ensembles of NV centers offer significant $\sqrt{N}$ ( $N$ is the number of color centers) improvement in the measurement precision when multiple centers probe the same parameter independently. For this reason, the concentration of $\mathrm{NV}$ centers [NV] and their spatial distribution within diamond matrix may be optimized for the specific sensing application, before reaching the regime of excessive defect density which ultimately deteriorates the sensitivity.

Although some NV centers and nitrogen atoms are always present in diamond, the NV concentration can be substantially increased by means of implanting additional nitrogen ions. A common alternative technique for improving the NV concentration is the irradiation of the sample with electrons [9], protons [10], or ions [11]. This is done to create vacancies in the lattice, which is followed by high-temperature $\left(>600^{\circ} \mathrm{C}\right)$ sample annealing that allows vacancies migration and recombination with the residual nitrogen present in the diamond. Importantly, the spatial- and ion-energy control of

\footnotetext{
* corresponding author; e-mail: mateusz.schabikowski@ifj.edu.pl
}

the implantation process enables tailoring of the local NV concentration towards specific applications.

In this work, we describe the preparation of approximately $20 \mu \mathrm{m}$ thin layers of NV centers by proton beam irradiation of a commercially available type-Ib diamond. Such thin sensor layers are useful for two-dimensional magnetic field imaging applications [12-14].

The implanted diamond samples, after annealing, were analyzed by means of the Raman spectroscopy, fluorescence, and optically detected magnetic resonance (ODMR). We have analyzed the NV creation efficiency and their spin decoherence as a function of the proton dose (fluence) in order to determine the optimum implantation parameters for sensing applications. Finally, the sample was annealed at $850^{\circ} \mathrm{C}$ for two hours in vacuum.

\section{Experiment}

The proton implantation was performed on a $3.0 \times 3.0 \times 0.3 \mathrm{~mm}^{3}$ sized type Ib diamond, synthesized by the high-pressure high-temperature (HPHT) technique. The sample was purchased from Element Six and has (100)-oriented main surfaces of which one is polished. The initial nitrogen concentration was $\left[\mathrm{N}_{i}\right] \lesssim 100 \mathrm{ppm}$.

Protons were implanted on the polished side of the sample using the ion beam from a Van de Graaff accelerator (located in the Institute of Nuclear Physics, Polish Academy of Sciences, Kraków, Poland) at the energy of $1.8 \mathrm{MeV}$ with the spot of approximately $20 \mu \mathrm{m}$ diameter. The proton beam was raster-scanned in order to implant $\sim 160 \mu \mathrm{m} \times 160 \mu \mathrm{m}$ square areas with controlled exposure time per pixel. The applied doses are listed in Table I. Numerical simulations performed with the SRIM software package [15] indicated that approximately 7 vacancies are created per proton and the penetration depth is nearly $20 \mu \mathrm{m}$. 


\section{TABLE I}

The list of proton-dose values of all implanted spots.

\begin{tabular}{c|c}
\hline \hline Spot \# & Dose $\left[\right.$ ions $\left./ \mathrm{cm}^{2}\right]$ \\
\hline 1 & $1.5 \times 10^{13}$ \\
2 & $1.5 \times 10^{14}$ \\
3 & $4.5 \times 10^{14}$ \\
4 & $1.5 \times 10^{15}$ \\
5 & $4.5 \times 10^{15}$ \\
6 & $1.5 \times 10^{16}$
\end{tabular}

After implantation, the sample was annealed for $2 \mathrm{~h}$ in an UHV system at the temperature of approximately $90{ }^{\circ} \mathrm{C}$ in order to increase the diffusion of vacancies and stimulate the formation of NV centers.

Photoluminescence (PL) of NV centers in diamond was observed in a home-made epifluorescence confocal microscope. Excitation was performed with a green $(532 \mathrm{~nm})$ laser light reflected from the dichroic mirror (DM). Fluorescence light was collected by the microscope objective $(10 \times, \mathrm{NA}=0.3)$ and, after passing DM and a long-pass optical filter (passband window: $600-800 \mathrm{~nm}$ ), was detected by a linear-mode avalanche photodiode. A motorized stage allowed to perform scans of the whole surface of samples.
ODMR signals were recorded using microwave (MW) field at around $2.87 \mathrm{GHz}$ delivered to the planar-ring antenna of a design similar to the one in Ref. [16], which was positioned around $0.5 \mathrm{~mm}$ above the sample. Along with the use of a high-power amplifier ( $45 \mathrm{~dB}$ gain), this allowed for a free sample movement while maintaining a high and stable MW field strength in the microscope focal point. The MW field was amplitude-modulated (100\% depth, $5 \mathrm{kHz}$ rate) and a phase-sensitive detection was performed with a lock-in amplifier to improve the signal-to-noise ratio.

\section{Results}

Before the implantation, we have ensured that the sample had negligible amount of NVs by performing fluorescence mapping (Fig. 1a) and, independently, by measuring the Raman spectra (not shown). Both methods consistently showed negligible (at the detection limit) initial and post-implantation (before annealing) NV concentrations. Nevertheless, Raman spectroscopy detected a low amount of NVs on the spot \#1 (the lowest dose; Fig. 2). However, we assumed the weak signal originated from the NVs present in the virgin sample (which is common for HPHT diamonds) and not those produced by the proton irradiation.
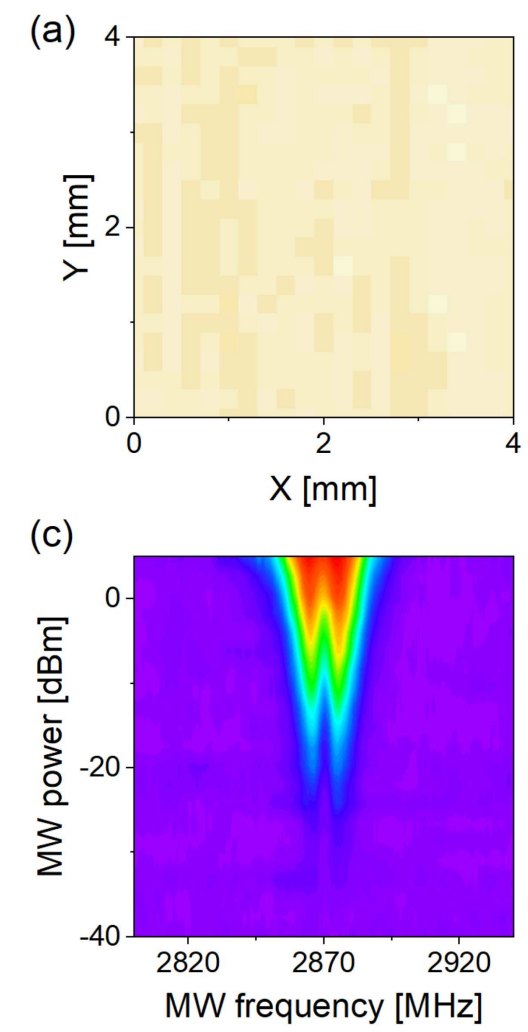

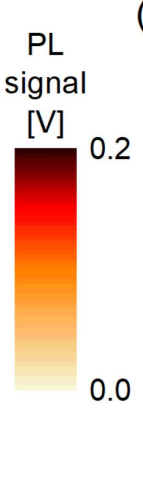

(b)

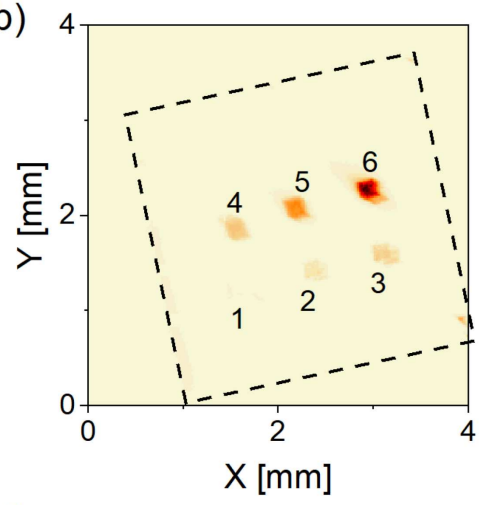

ODMR contrast

[\%]

(d)

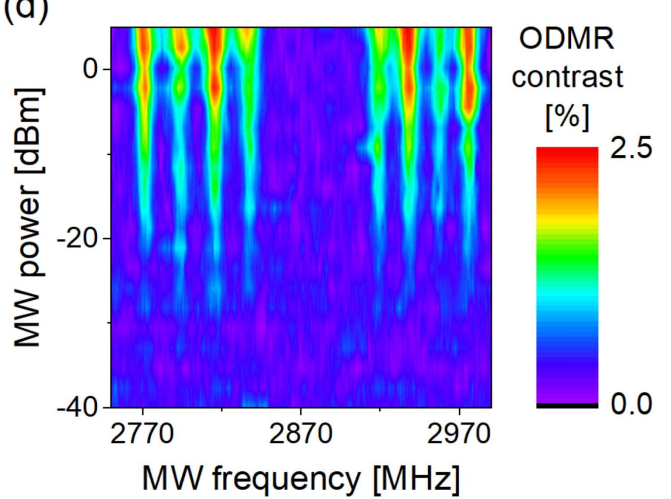

PL signal

[V]

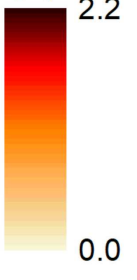

0.0

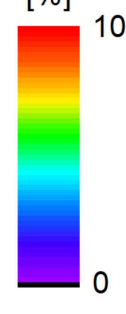

MW frequency $[\mathrm{MHz}]$

Fig. 1. Photoluminescence of the irradiated sample prior to (a) and after (b) the annealing. Numbers in (b) indicate the spots implanted according to doses listed in Table I, while the dashed square indicates the edges of the sample; ODMR contrast measured at spot \#6 as a function of MW power and frequency before the amplifier without (c) and with (d) applied magnetic field ( $\sim 5 \mathrm{mT}$, arbitrarily oriented). 


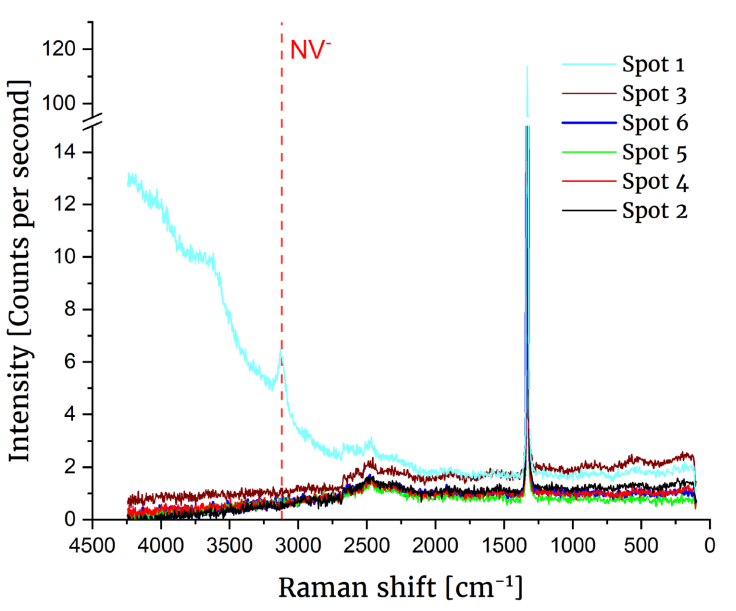

Fig. 2. Raman spectra of the irradiated sample prior to annealing. The red dashed line indicates the light wave number attributed to NVs.

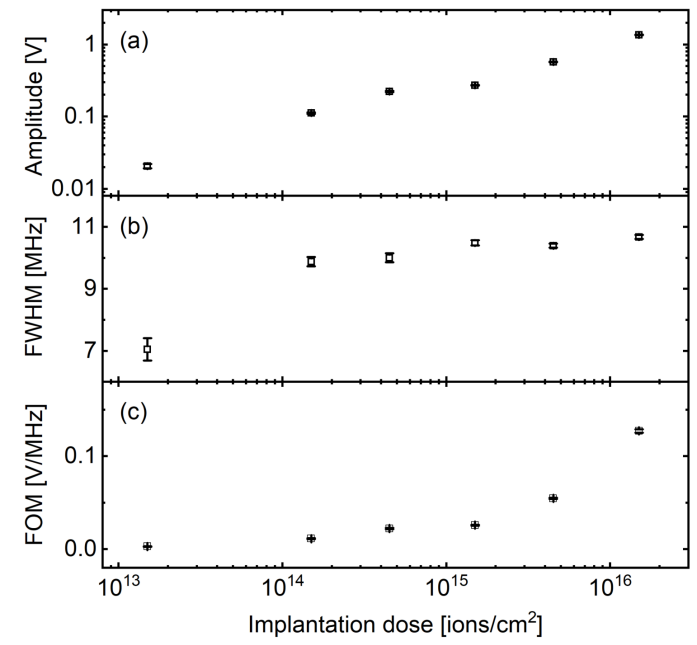

Fig. 3. The numerical analysis of the lowest-frequency ODMR resonances (see Fig. 1d) observed at low $(-30 \mathrm{dBm}) \mathrm{MW}$ power as a function of the implantation dose: (a) semi-log plot of peak amplitudes, (b) peak widths (FWHM), and (c) the figure of merit for magnetometry applications (amplitude over width). The error bars represent fit uncertainty.

In order to quantitatively evaluate the effect of proton implantation on spin properties of the formed NV centers, we analyzed the properties of ODMR at low MW power $(\leq-30 \mathrm{dBm}$ in Fig. 1d) in order to avoid any power-broadening by the MW field. For each spot, the ODMR spectrum was taken with the help of the lockin amplifier, averaged (25-64 times), and a Gaussian fit was applied to the lowest-frequency resonance (the leftmost peak in Fig. 1d). The result of this analysis is shown in Fig. 3.

Figure 3a depicts ODMR signal amplitudes recorded with the lock-in for all applied implantation doses. Such signal is proportional to the fluorescence of NV centers, hence, to their concentration, $[\mathrm{NV}]$, in diamond. It continuously increases with the growing dose.
The growth enhances optical signal and improves the detection signal-to-noise ratio, which is favorable for sensing applications. Moreover, the lack of visible saturation of the growth trend hints at the possibility of further signal improvements for even larger implantation doses. The initial comparison of fluorescence levels for spot \#6 with our diamond samples prepared by electron irradiation allows us to estimate the NV concentration to be in the range of $0.1-1 \mathrm{ppm}[17]$.

The width of the ODMR resonance is governed by the NVs spin-dephasing rate. For type Ib diamonds, due to the high nitrogen content and the resulting inhomogeneous broadening, the hyperfine structure of NVs is not resolved and the linewidth becomes Gaussian with a width of $\gtrsim 5 \mathrm{MHz}$. Figure $3 \mathrm{~b}$ shows the measured ODMR peak widths in the implanted diamond sample. Apart from the jump at the lowest dose, which we attribute to the fit quality for the weakest observed signal, the widths gradually increase with the increase of dose. We attribute it to radiation damage in the crystal lattice similar to the observed in electron implanted samples [18].

The sensitivity of dc and low-frequency magnetometers based on NV centers is fundamentally limited by the spin decoherence rate of the NV centers, i.e., the reciprocal of their dephasing time. With optical detection it is reduced due to the low contrast of ODMR resonances and limited collection efficiency of fluorescence photons. When the number of PL photons is relatively low, the optical shot-noise may be a dominating source of noise. However, in many experimental realizations the noise level is dominated by the detectors and electronics used, hence, the signal-to-noise ratio improves with the increasing signal amplitude. These constraints are conveniently illustrated by figure of merit (FOM) defined as a peak amplitude over its width (Fig. 3c).

FOM combines both the negative and positive effects (increase of the peak width and the amplitude, respectively) of increasing irradiation doses. Despite the increasing radiation damage at larger doses, the positive effect of the growing amplitude dominates over the whole range of applied doses without reaching a saturation point (at which the FOM values would start to decrease). This result suggests that the dose can be further increased to improve the sensitivity of similar sensors.

\section{Conclusions}

We have demonstrated the efficient creation of dense, $20 \mu \mathrm{m}$ thick layers of nitrogen vacancies in diamond crystals using proton implantation and UHV annealing. The results are consistent with numerical simulations of the crystal damage and the initial nitrogen content. The obtained concentration of NV centers is of the order of $[\mathrm{NV}] \sim 0.1--1 \mathrm{ppm}$ for $10^{16}$ protons $/ \mathrm{cm}^{2}$ and our results indicate that it may be further improved by increasing the dose, resulting in high-quality NV diamond samples for bio-magnetic sensing applications. 


\section{Acknowledgments}

The authors are particularly grateful to Dr. Janusz Lekki for the proton implantation. The authors also acknowledge Karol Cieślik and Prof. Franciszek Krok for annealing the sample. This work was financially supported by the DIABASE (ERA-NET Rus + Programme), the $2016 / 21 / B / S T 7 / 01430$ (Polish National Science Center, NCN) grant and the MNiSW 7150/E-338/M/2018 grants.

\section{References}

[1] M.W. Doherty, N.B. Manson, P. Delaney, F. Jelezko, J. Wrachtrup, L.C.L. Hollenberg, Phys. Rep. 528, 1 (2013).

[2] R. Schirhagl, K. Chang, M. Loretz, C.L. Degen, Ann. Rev. Phys. Chem. 65, 83 (2013).

[3] T. Unden, P. Balasubramanian, D. Louzon, et al., Phys. Rev. Lett. 116, 230502 (2016).

[4] L. Rondin, J.P. Tetienne, T. Hingant, J.F. Roch, P. Maletinsky, V. Jacques, Rep. Progr. Phys. 77, 056503 (2014).

[5] T. Wolf, P. Neumann, K. Nakamura, H. Sumiya, T. Ohshima, J. Isoya, J. Wrachtrup, Phys. Rev. X $\mathbf{5}$ 041001 (2015).

[6] P. Neumann, I. Jakobi, F. Dolde, C. Burk, R. Reuter, G. Waldherr, J. Honert, T. Wolf, A. Brunner, J.H. Shim, D. Suter, H. Sumiya, J. Isoya, J. Wrachtrup, Nano Lett. 13, 6 (2013).

[7] G. Balasubramanian, I.Y. Chan, R. Kolesov, et al., Nature 455, 648 (2008).
[8] P. Maletinsky, S. Hong, M.S. Grinolds, B. Hausmann, M.D. Lukin, R.L. Walsworth, M. Loncar, A. Yacoby, Nature Nanotechnol. 7, 320 (2012).

[9] C.A. McLellan, B.A. Myers, S. Kraemer, K. Ohno, D.D. Kenichi, D.D. Awschalom, A.C. Bleszynski Jayich, Nano Lett. 16, 2450 (2016).

[10] J. Botsoa, T. Sauvage, M.P. Adam, P. Desgardin, E. Leoni, B. Courtois, F. Treussart, M.F. Barthe, Phys. Rev. B 84, 125209 (2011).

[11] I. Aharonovich, C. Santori, B.A. Fairchild, J. Orwa, K. Ganesan, K.M.C. Fu, R.G. Beausoleil, A.D. Greentree, S. Prawer, J. Appl. Phys. 106, 1 (2009).

[12] L.M. Pham, D. Le Sage, P.L. Stanwix, T.K. Yeung, D. Glenn, A. Trifonov, P. Cappellaro, P.R. Hemmer, M.D. Lukin, H. Park, A. Yacoby, R.L. Walsworth, New J. Phys. 13, 045021 (2011).

[13] A.M. Wojciechowski, M. Karadas, A. Huck, C. Osterkamp, S. Jankuhn, J. Meijer, F. Jelezko, U.L. Andersen, Rev. Sci. Instrum. 89, 031501 (2018.

[14] M. Karadas, A.M. Wojciechowski, A. Huck, N.O. Dalby, U.L. Andersen, A. Thielscher, Sci. Rep. 8, 4503 (2018).

[15] J.F. Ziegler, M.D. Ziegler, J.P. Biersack, Nucl. Instrum. Methods Phys. Res. B 268, 1818 (2010).

[16] K. Sasaki, Y. Monnai, S. Saijo, R. Fujita, H. Watanabe, J. Ishi-Hayase, K.M. Itoh, E. Abe, Rev. Sci. Instrum. 87, 053904 (2016).

[17] M. Mrózek, D. Rudnicki, P. Kehayias, A. Jarmola, D. Budker, W. Gawlik, EPJ Quant. Technol. 2, 22 (2015).

[18] E. Kim, V.M. Acosta, E. Bauch, D. Budker, R.P. Hemmer, Appl. Phys. Lett. 101, 082410 (2012). 